\title{
Traditional Chinese Medicine Tang-Luo-Ning Ameliorates Sciatic Nerve Injuries in Streptozotocin-Induced Diabetic Rats
}

\author{
Da-Wei Zou, ${ }^{1}$ Yan-Bin Gao, ${ }^{1}$ Zhi-Yao Zhu, ${ }^{1}$ Hui Zhou, ${ }^{2}$ Tao-Jing Zhang, ${ }^{2}$ Bu-Man Li, ${ }^{3}$ \\ Jin-Yang Wang, ${ }^{1}$ Min-Zhou Li, ${ }^{2}$ Ming-Fei Ma, ${ }^{2}$ and Na Zhang ${ }^{1}$ \\ ${ }^{1}$ School of Traditional Chinese Medicine, Capital Medical University, Number 10 Youanmenwai Xitoutiao, Fengtai District, \\ Beijing 100069, China \\ ${ }^{2}$ Endocrinology Department, Oriental Hospital of Beijing University of Chinese Medicine, Beijing, China \\ ${ }^{3}$ Traditional Chinese Medicine Department, Shougang Hospital of Peking University, Beijing, China
}

Correspondence should be addressed to Yan-Bin Gao; gaoyb111@hotmail.com

Received 16 July 2013; Accepted 1 September 2013

Academic Editor: Vincenzo De Feo

Copyright (c) 2013 Da-Wei Zou et al. This is an open access article distributed under the Creative Commons Attribution License, which permits unrestricted use, distribution, and reproduction in any medium, provided the original work is properly cited.

\begin{abstract}
Diabetic peripheral neuropathy (DPN) is a common microvascular complication of diabetes associated with high disability rate and low quality of life. Tang-Luo-Ning (TLN) is an effective traditional Chinese medicine for the treatment of DPN. To illustrate the underlying neural protection mechanisms of TLN, the effect of TLN on electrophysiology and sciatic nerve morphology was investigated in a model of streptozotocin-induced DPN, as well as the underlying mechanism. Sciatic motor nerve conduction velocity and digital sensory nerve conduction velocity were reduced in DPN and were significantly improved by TLN or $\alpha$-lipoic acid at 10 and 20 weeks after streptozotocin injection. It was demonstrated that TLN intervention for 20 weeks significantly alleviated pathological injury as well as increased the phosphorylation of ErbB2, Erk, Bad (Ser112), and the mRNA expression of neuregulin 1 (Nrg1), GRB2-associated binding protein 1 (Gabl), and mammalian target of rapamycin (Mtor) in injured sciatic nerve. These novel therapeutic properties of TLN to promote Schwann cell survival may offer a promising alternative medicine for the patients to delay the progression of DPN. The underlying mechanism may be that TLN exerts neural protection effect after sciatic nerve injury through $\mathrm{Nrg1} / \mathrm{ErbB} 2 \rightarrow$ Erk/Bad Schwann cell survival signaling pathway.
\end{abstract}

\section{Introduction}

Diabetic peripheral neuropathy (DPN) is a common microvascular complication of diabetes and affects more than $50 \%$ of diabetic patients [1]. DPN is a progressive disease characterized by pain and paresthesia and can even develop diabetic foot or gangrene [2]. Recent studies showed that the incidence of DPN is a complex mechanism mainly including (1) hyperglycemia and metabolism disorders such as activation of polyol pathway [3], nonenzymatic glycosylation [4], and activation of protein kinase C [5]; (2) activation of mitogen-activated protein kinases (MAPK) pathways [6]; (3) oxidative stress [7]; (4) lack of neurotrophic support and vascular pathologies [8]; (5) apoptosis of Schwann cells (SCs) [9].
As an important neuron in peripheral nerve system, SCs have become a center for many research studies in recent years as they play a key role in myelination, neurotrophic support, homeostasis, and repair after peripheral nerve injury [10-13]. Moreover, physiological interactions and reciprocal signaling between axons and SCs play critical roles in nerve development and survival, and $\mathrm{Nrgl}$ is an essential modulator of SCs during development, nerve repair, and remyelination [14]. However, conventional therapy techniques for DPN, including controlling hyperglycemia, improving microcirculation, alleviating metabolic disorder, and suppressing oxidative stress, have failed to halt functional and structural changes of peripheral nerve in both humans and experimental animals [15]. Significantly, this suggests that the injuries of SCs have been activated by hyperglycemia induced 
metabolic disturbance, and then SCs can act independently to further exacerbate the structural and functional injuries of sciatic nerve. The therapies above could not reverse the occurred peripheral nerve injuries such as axonal degeneration, demyelination, and cell apoptosis. Consequently, improvement of SCs survival and recovery of peripheral nerve regeneration ability are of vital importance to delay the progress of DPN.

As is well known, the incidence of DPN could be attributed to a multiple mechanisms. Traditional Chinese medicine (TCM), which has the advantage of hitting multiple targets at the same time, may be a good choice for such therapeutic agents [16]. Tang-Luo-Ning (TLN) is an effective Chinese recipe in TCM for the treatment of DPN $[17,18]$. According to the theory of TCM, TLN recipe mainly contains three different traditional Chinese herbs: Astragalus membranaceus (Fisch.) Bge (Huangqi), Salvia miltiorrhiza Bge. (Danshen), and Paeonia lactiflora Pall. (Chishao) (detailedly shown in Section 2). This recipe functions to tonify qi promote the blood circulation and remove the blood stasis. It has been reported that Astragalus membranaceus could reduce the serum glucose and triglyceride level and inhibit advanced glycation end products-induced inflammation as well in the diabetic individuals [19, 20]. Moreover, Astragaloside IV, the major active component of TLN recipe, could exert protective effects against DPN in streptozotocin- (STZ-) induced diabetes in rats through several interrelated mechanisms [21]. Previously, we demonstrated that TLN exerted protective effects against the progression of DPN through several interrelated mechanisms, such as improvement of oxidative damage, inhibition of dorsal root ganglion and sciatic nerve apoptosis [22, 23] in streptozotocin-induced diabetic rats (STZ-R). However, whether TLN intervention attenuates sciatic nerve injury and promotes recovery of it through enhancing SCs survival is unknown.

In our study, due to multiple-target characteristic of Chinese herbal compound, microarray expression analysis was used to screen cell-survival related genes and pathways regulated by TLN, followed further validation by quantitative real time polymerase chain reaction ( $\mathrm{qPCR}$ ) and western blot.

\section{Materials and Methods}

2.1. The TLN Extract Preparation and Quality Control. TLN recipe mainly contains the ingredients from three different traditional Chinese herbs: the dried root of Astragalus membranaceus (Fisch.) Bge. (Huangqi, amount used $30 \mathrm{~g}$ per day), the dried root and rhizome of Salvia miltiorrhiza Bge. (Danshen, amount used $30 \mathrm{~g}$ per day), and the dried root of Paeonia lactiflora Pall. (Chishao, amount used $20 \mathrm{~g}$ per day) as well. All herbs were purchased from Oriental Hospital of Beijing University of Chinese Medicine (BUCM, Beijing, China). The crude drugs were carefully authenticated and then decocted and made into particle in the Manufacturing Laboratory of Oriental Hospital of BUCM. In brief, the weight proportion of Astragalus membranaceus (Fisch.) Bge., Salvia miltiorrhiza Bge., and Paeonia lactiflora Pall. is $3: 3: 2$. The herbs were extracted three times with $60 \%$ ethanol
(600 mL per $100 \mathrm{~g}$ herbs) for $1.5 \mathrm{~h}$ each time. The ethanol extract was pooled and concentrated to stronger liquor with a relative density of $1.25-1.30$ at $70^{\circ} \mathrm{C}$. The above liquor was evaporated under reduced pressure and ground into fine powder to obtain the TLN extract. The TLN extract and excipients were mixed at a ratio of $1: 1$ with $80 \%$ ethanol to pelletize. A content of $0.7875 \mathrm{mg} / \mathrm{g}$ Astragaloside IV, which is the main active component in TLN, was detected in TLN particles by HPLC-ELSD (shown in Supplementary Figure 1, available on line at http://dx.doi.org/10.1155/2013/989670). TLN particles were suspended in the distilled water at appropriate concentration for the animal treatment.

\subsection{Animal Model and Drug Intervention. Male Sprague} Dawley rats weighting $180 \mathrm{~g}-220 \mathrm{~g}$ were purchased from Vital River Laboratories (Beijing, China, certificate No. SCXK 2006-0009). The animals were housed in separate cages at constant temperature $\left(20-22^{\circ} \mathrm{C}\right)$ and humidity $(50 \%-67 \%)$ under a 12-hour light/dark cycle in specific pathogen free animal laboratory of BUCM. The animals had free access to standard chow diet (Vital River, Beijing, China) and sterilized drinking water during the period of this experiment. The experimental protocol was approved by the ethics committee of BUCM. All animal experiments were conducted in accordance with the NIH guide for the care and use of laboratory animals (NIH Publication No. 80-23; revised 1978).

After one week of acclimatization, rats were given STZ (Sigma, $60 \mathrm{mg} / \mathrm{kg}$ body weight), and 72 hours later rats with fasting blood glucose more than $16.7 \mathrm{mmol} / \mathrm{L}$ were considered successfully induced for diabetes. The diabetic rats were randomly divided into three groups including STZ induced diabetic group (STZ group), Tang-Luo-Ning group (TLN group), and alpha-lipoic acid group ( $\alpha$-LA group); there was also a non-STZ induced group as control group (CTL group). Rats in intervention groups were administrated separate drug by gavage $(5 \mathrm{~mL} / \mathrm{kg})$ from the next day at a dose of either $10 \mathrm{~g} / \mathrm{kg}$ body weight (TLN group) or $20 \mathrm{mg} / \mathrm{kg}$ body weight ( $\alpha$-LA group), and the rats in CTL group or STZ group were treated at the same volume of distill water $(5 \mathrm{~mL} / \mathrm{kg})$ by gavage for 20 weeks. The $\alpha$-LA was purchased from Puritan's Pride Inc. (Oakdale, NY, USA). The batch number of the $\alpha$ LA used in this experiment was 250597-09. Determination of the dose of TLN used in the present study was based on our previous works. After the last administration, all rats were fasted for 12 hours before experiment but allowed free access to water. The animals were anesthetized using $10 \%$ chloral hydrate $(300-350 \mathrm{mg} / \mathrm{kg})$ to get sciatic nerve. The sciatic nerve tissue from each mouse was also divided into two parts, one part was immediately frozen in liquid nitrogen for western blot, RNA extraction, and microarray, and another part was fixed with $4 \%$ glutaraldehyde for electron microscope, respectively.

2.3. Electrophysiological Measurements. Sciatic motor nerve conduction velocity (MNCV) and distal digital sensory nerve conduction velocity (SNCV) were measured after induction of anesthesia with $10 \%$ chloral hydrate $(300-350 \mathrm{mg} / \mathrm{kg})$ at two points (10 weeks and 20 weeks after intervention). Body 

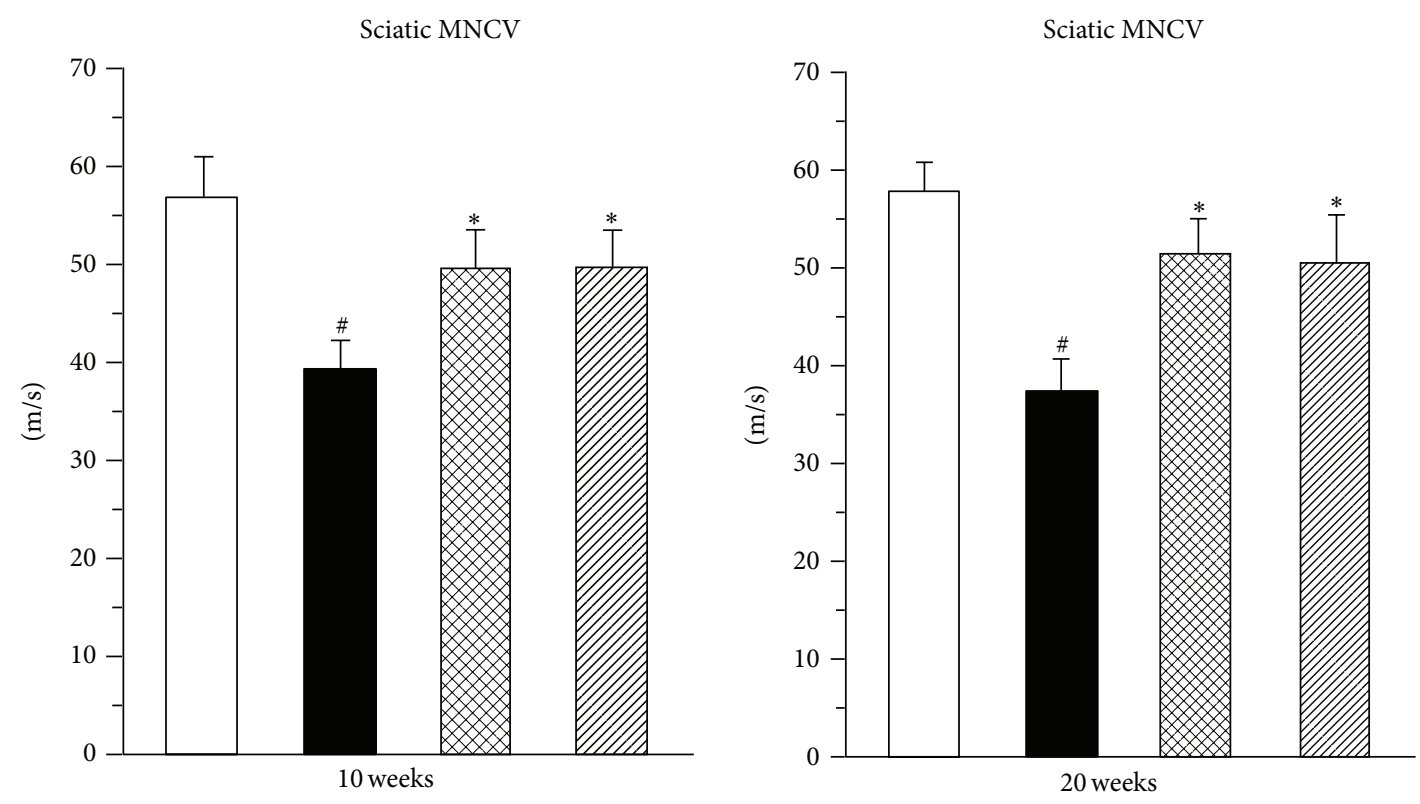

(a)
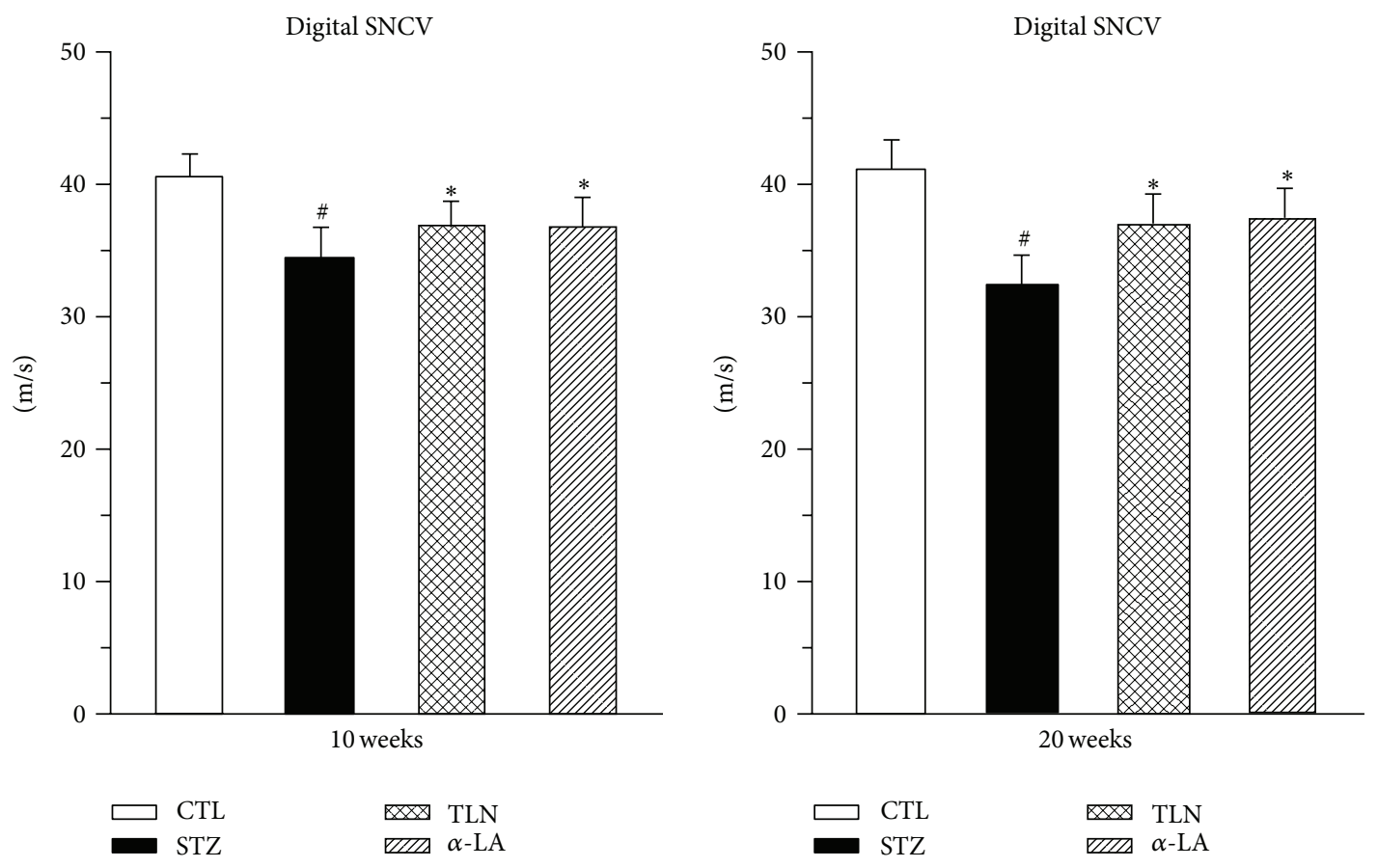

(b)

FIgURE 1: Effects of TLN and $\alpha$-LA on (a) sciatic MNCV and (b) distal digital SNCV at 10 and 20 weeks after streptozotocin injection. $n=8$ per group. Data are expressed as mean \pm SD. ${ }^{\#} P<0.05$ versus CTL group, ${ }^{*} P<0.05$ versus STZ group; sciatic MNCV and distal digital SNCV of STZ group rats with streptozotocin injection decreased compared with CTL group $(P<0.05)$. Tang-Luo-Ning and alpha-lipoic acid intervention both increased sciatic MNCV and distal digital SNCV of injured sciatic nerve compared with STZ group $(P<0.05)$. MNCV: Motor nerve conduction velocity. SNCV: sensory nerve conduction velocity. Four groups: CTL group: nonstreptozotocin-induced group, STZ group: streptozotocin-induced diabetic group, TLN group: Tang-Luo-Ning group, and $\alpha$-LA group: alpha-lipoic acid group.

temperature was maintained at $37^{\circ} \mathrm{C}$ with a warming pad. For sciatic measurement, the left sciatic motor conduction system was stimulated at the sciatic notch where sciatic nerve exits and at ipsilateral ankle via bipolar electrodes with a width of
$0.1 \mathrm{~ms}$. The threshold was determined when the compound muscle action appeared or disappeared, and we set at 1.5fold above the threshold as stimulus intensity. The recorded bipolar electrodes were placed at the first interosseous muscle 
of the hind-paw, as well as reference electrode was placed $1 \mathrm{~cm}$ away from the recording electrode but between the stimulating and the recording electrode. Hind-limb SNCV was recorded in the digital nerve to the second toe by stimulating with a square-wave pulse duration of $0.05 \mathrm{~ms}$ using the smallest intensity current that resulted in a maximal amplitude response. The sensory nerve action potential was recorded behind the medial malleolus. The maximal SNCV was calculated from the latency to the onset of the initial negative deflection and the distance between stimulating and recording electrodes. The latencies of action potentials were measured as described below by physiological data recording system (MacLab/400, ADI, Australia) and dual-beam memory oscilloscope (VC-10, Nihon Kohden Corporation, Japan). Average conduction time was calculated after 7-10 measurements. Sciatic MNCV and distal digital SNCV were measured and calculated based on the method [24].

2.4. Morphological Observation of the Sciatic Nerve. Approximately $0.1 \mathrm{~cm}$ of sciatic nerve was removed from the lower edge of the left piriformis and cut into pieces about $1 \mathrm{~mm}^{3}$ by volume. After prefixing with cold, $4 \%$ glutaraldehyde nerve tissues were fixed with $1 \%$ osmic acid and dehydrated and embedded in 1:100 mixture of Epon 812 and 100\% aceton. Semithin sections were cut and stained with toluidine blue before they were further cut into $50 \mathrm{~nm}$ ultrathin sections using an ultramicrotome (8800, LKB, Bromma, Sweden). After double staining with uranyl acetate and lead nitrate, morphological changes of the sciatic nerves were observed by transmission electron microscopy (H-600, Hitachi, Japan). Images were taken by imaging systems (Moticam 2306, Motic Instruments Inc., Canada).

2.5. RNA Extraction and Microarray. RNA extraction and microarray were performed according to manufacturer's protocols by Shanghai Biotechnology Co., Ltd. TRIZOL Reagent (Catalogue number 15596-018, Life technologies, Carlsbad, CA, USA) was added into sciatic nerve, and total RNA was isolated using RNeasy Kit (Qiagen). RNA integrity number (RIN) and $28 \mathrm{~s} / 18 \mathrm{~s}$ ratio were determined by Agilent BioAnalyzer 2100. RNAs with RIN $\geq 7.0$ and $28 \mathrm{~S} / 18 \mathrm{~S}>0.7$ were deemed to be of sufficient quality. RNA concentration and A260/A280 ratio were determined by Nanodrop ND1000. RNAs were purified by QIAGEN RNeasy Kit, followed by cDNA synthesis and cRNA fluorescent labeling, purifying, and shearing. Probes were hybridized with Rat Gene Expression Microarray slides $(4 \times 44 \mathrm{~K}$ microarray, Agilent, Catalogue number G2519F-014879) in a hybridization oven (Agilent G2545A). After thorough washing, array slides were scanned by Agilent scanner with both $100 \%$ and $10 \%$ PMT setting. Results were analyzed by Agilent software.

2.6. Microarray Data Analysis. With threshold of fold change between experimental samples (TLN group) and control samples (STZ group, CTL group) set at 2, genes that were differentially regulated by TLN were identified. Genes that were first downregulated $>2$-fold (compared with CTL) by STZ and then upregulated $>2$-fold (compared with STZ) by TLN were considered as upregulated genes, and the downregulated genes were opposite. Differentially regulated genes by TLN were analyzed using an online SAS system for hierarchical clustering, Gene ontology (GO) enrichment analysis and Kyoto Encyclopedia of Genes and Genomes pathway analysis (enrichment $P<0.05$ ).

2.7. $q P C R$. To confirm microarray data and further screen cell survival related pathway, Nrg1, GRB2-associated binding protein 1 (Gab1), mammalian target of rapamycin (Mtor), and phosphoinositide-3-kinase, catalytic, beta polypeptide (Pik3cb) were selected for validation by qPCR. Primers were designed by Sheng Gong Biotech (Shanghai, China) using Primer Premier 5.0 software (PREMIER Biosoft International, CA, USA). The sequences of Nrg1, Pik3cb, Mtor, Gabl, and GAPDH were the following: Nrgl: forward primer $5^{\prime}$-TGGCACATCCATCCAAATAC-3', reverse primer $5^{\prime}$-GTAGCATGCTGCTGGGTCTA-3'; Pik3cb: forward primer $5^{\prime}$-AGATGTTGCTCAGCTTCAGG-3', reverse primer $5^{\prime}$-TT CATCACTCATCTGTCGCA-3 ${ }^{\prime}$; Mtor: forward primer $5^{\prime}$-AGAACCACATGCCACACAGT$3^{\prime}$, reverse primer $5^{\prime}$-CTTTGGCATTTGTGTCCATC-3'; Gabl: forward primer $5^{\prime}$-CTCCTGAGACCACAAAGCAA3', Reverse primer $5^{\prime}$-AACGCTAGCTGCTTCTCACA-3'; GAPDH: Forward primer $5^{\prime}$-CAACTCCCTCAAGATTGTCAGCAA-3', Reverse primer: $5^{\prime}$-GGCATGGACTGTGGTCATGA-3'. RNA was reverse transcribed by M-MLV Reverse Transcriptase (CK2801A, Takara) according to manufacturer's protocols, and $\mathrm{qPCR}$ was performed on qPCR machine (ABI 7500, Life Technologies, USA) with the following program settings: $94^{\circ} \mathrm{C}$ for $15 \mathrm{~min}, 94^{\circ} \mathrm{C}$ for $15 \mathrm{~s}$, $60^{\circ} \mathrm{C}$ for $34 \mathrm{~s}$, and $72^{\circ} \mathrm{C}$ for $15 \mathrm{~s}$ for 40 cycles followed by $72^{\circ} \mathrm{C}$ for $10 \mathrm{~min}$. All qPCRs were performed in duplicate, and relative expression was calculated using the $2^{-\Delta \Delta \mathrm{CT}}$ method [25].

2.8. Western Blot. To determine the expression level of (P-) ErbB2, extracellular signal-regulated kinase (Erk), PErk, protein kinase B (PKB/Akt), P-Akt, bad, and P-bad Ser112/Ser136, the sciatic nerve tissues were quickly pulverized in prechilled mortar and then fractionated by SDSPAGE. $40 \mu \mathrm{g}$ of protein was separated by electrophoresis and transferred to Nitrocellulose membrane (Millipore, USA). Membrane was blocked in 5\% nonfat dry milk in Trisbuffered saline (containing 0.1\% Tween-20, $\mathrm{PH}=7.6$ ) for $1 \mathrm{~h}$ and incubated with primary antibodies at $4^{\circ} \mathrm{C}$ overnight. The primary antibodies were as follows: mouse monoclonal antiErk1/2 antibody (dilution: 1:3000), mouse monoclonal antiPhospho-Erk1/2 antibody (dilution: 1:2000), rabbit monoclonal anti-Bad (D24A9) antibody (dilution: 1:2000), rabbit monoclonal anti-Phospho-Bad (Ser112) (40A9) antibody (dilution: 1:500), rabbit monoclonal anti-PhosphoBad (Ser136) (D25H8) antibody (dilution: 1:500), rabbit monoclonal anti-Akt antibody (dilution: 1:3000), and rabbit monoclonal anti-Phospho-Akt (Ser473) antibody (dilution: 1:2000); the antibodies above were all purchased from Cell Signaling Technology (USA). Rabbit polyclonal antiErbB2 antibody (dilution: 1:4000, Novus, Biologicals, Inc.; Littleton, CO, USA), Rabbit polyclonal anti-Phospho-ErbB2 


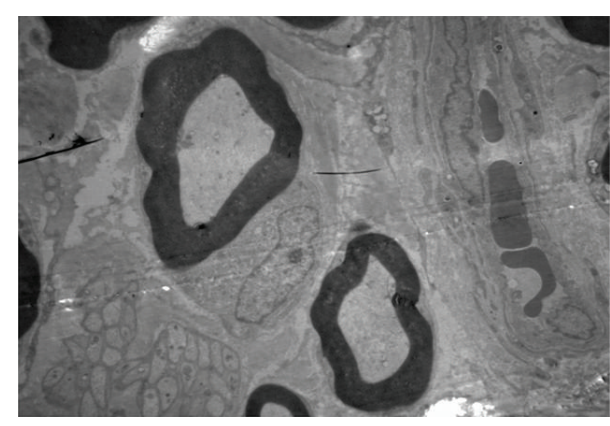

(a)

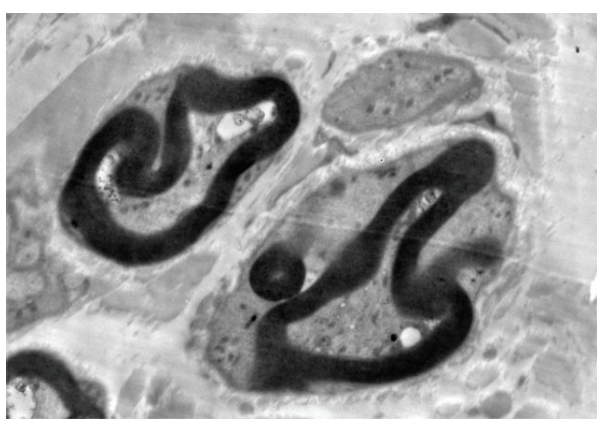

(c)

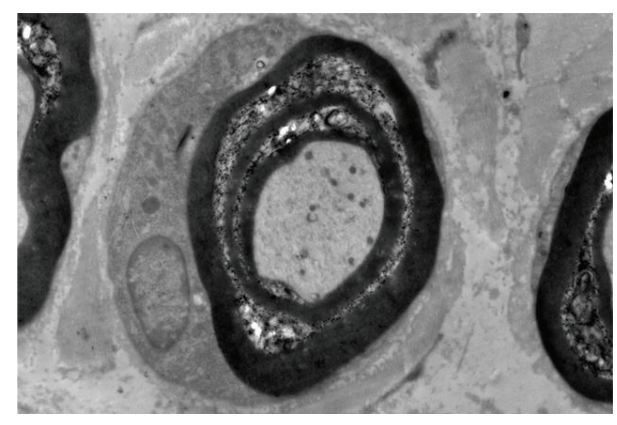

(b)

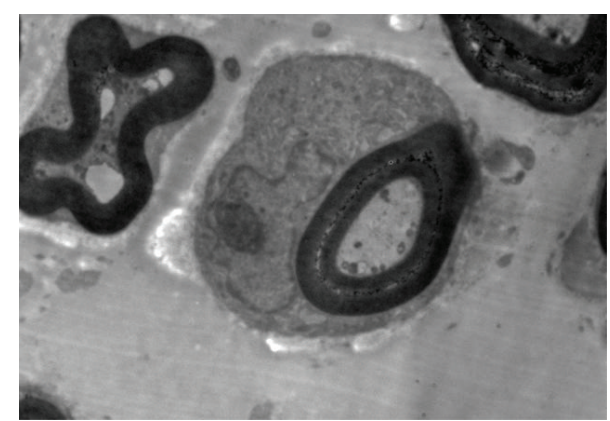

(d)

FIGURE 2: Transmission electron micrographs showing the ultrastructure of sciatic nerve fiber $(\times 10000)$ (a) CTL rats: intact myelinated axon. (b) STZ rats: serious demyelination, axial degeneration, and Schwann cell proliferation. (c) TLN rats: moderate segmental demyelination and axial degeneration. (d) $\alpha$-LA rats: moderate segmental demyelination, axial degeneration, and Schwann cell proliferation. Four groups: CTL group: nonstreptozotocin-induced group, STZ group: streptozotocin-induced diabetic group, TLN group: Tang-Luo-Ning group, and $\alpha$-LA group: alpha-lipoic acid group.

(Tyr1248) antibody (dilution: 1:3000, Novus, Biologicals, Inc.; Littleton, CO, USA), after washing $3 \times 10$ min with Trisbuffered saline, horseradish peroxidase conjugated secondary antibody (dilution 1:1000: P-Bad ser112/ser136, 1:2000: other antibodies) was added and incubated for $1 \mathrm{hr}$ with shaking. Target proteins were visualized using enhanced chemiluminescence reagents and exposed to X-Ray film in dark room. Densitometry analysis was performed on protein bands using software IPP 6.0 (Media Cybernetics Inc., USA).

2.9. Statistical Analysis. Differential gene expression and bioinformatics analysis were performed using online SAS analysis system provided by Shanghai Biotechnology Corporation. All other data were statistically analyzed by SPSS13.0 software. Average value \pm standard deviation (Mean \pm SD) were calculated, one-way ANOVA (analysis of variance) and LSD- $t$ test (least significant difference $t$ test) for multiple samples multiple comparison were performed, and $P<0.05$ were considered as statistically significant.

\section{Results}

\subsection{Function and Morphology of Sciatic Nerve}

3.1.1. TLN Increases Nerve Conduction Velocity in STZInduced Diabetic Rats. Compared with the STZ group, an obvious improvement of sciatic MNCV and distal digital SNCV were observed after 10 weeks of TLN treatment
(Figure 1), and this trend was even more profound after 20 weeks of TLN treatment. Moreover, the trend of MNCV after $\alpha$-LA treatment was consistent with published data [26]. In brief, results demonstrated that TLN intervention increased MNCV and distal digital SNCV of injured sciatic nerve and improved the lower nerve function.

3.1.2. TLN Alleviates Pathological Injury of Sciatic Nerve. In control rats, there were fair-arranged nerve fibers, mainly with myelinated nerve of thick myelin and large axon diameter simultaneously. In diabetic rats, serious demyelination, axial degeneration and reduced number of myelinated nerve fibers were observed on transmission electron microscope, and the pathological injury in this study corresponded to typical morphological changes of DPN. Likewise, the pathologic and morphological changes in the sciatic nerves of diabetic rats treated with TLN and $\alpha$-LA were both greatly alleviated (Figure 2). No significant changes were observed in the number and proportion of myelinated and unmyelinated nerve fibers in TLN and $\alpha$-LA group. The results demonstrated that TLN could be counteracting against the pathological morphology changes of sciatic nerve in STZ-induced diabetic rats.

\subsection{Schwann Cells Survival of Sciatic Nerve}

3.2.1. Microarray Bioinformatics Analysis of Differentially Regulated Genes by TLN. Using an online SAS system, cluster 


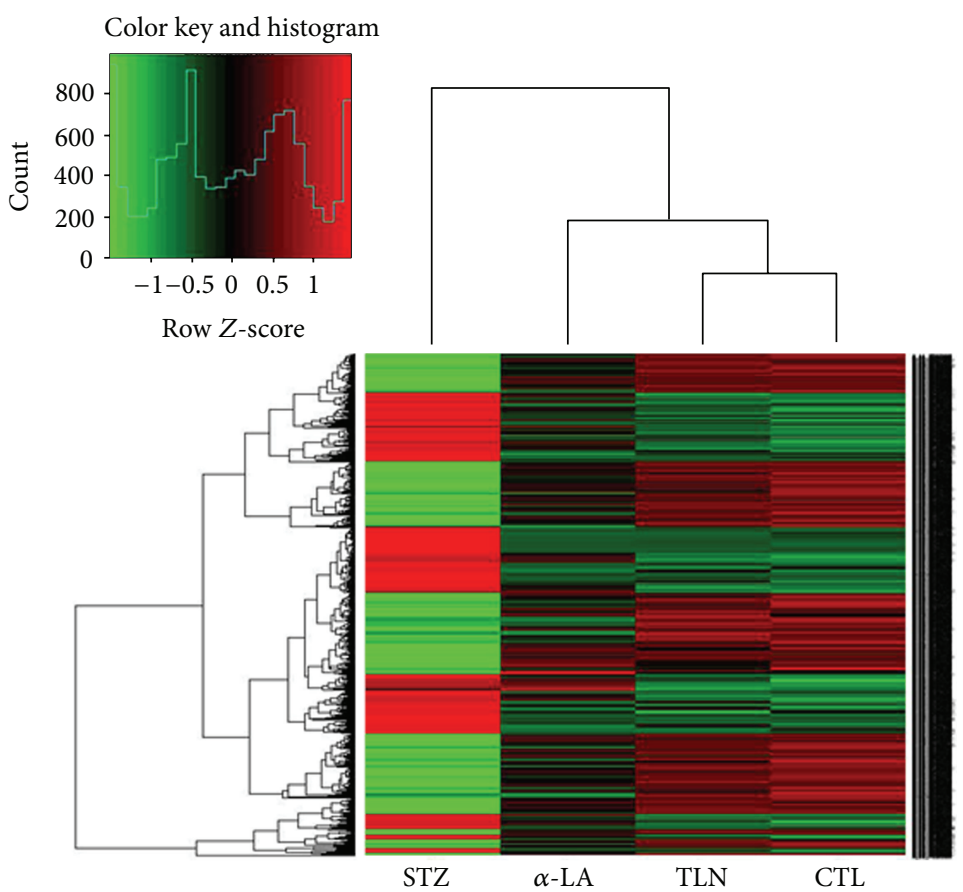

FIGURE 3: Heat map showed the differentially expressed genes regulated by TLN between four groups. Red color indicates over expressed genes, whereas green color indicates the opposite. The color scale bar is shown. Four groups: CTL group: nonstreptozotocin-induced group, STZ group: streptozotocin-induced diabetic group, TLN group: Tang-Luo-Ning group, and $\alpha$-LA group: alpha-lipoic acid group.

analysis was performed and the resultant heat map allowed us to visualize the differential gene expression patterns between the experimental groups (Figure 3). GO analysis encompasses three domains: molecular function, biological process, and cellular component. GO analysis showed that the functions of genes that were differentially regulated by TLN mainly focused on cellular homeostasis, cell development, and anatomical structure formation (Figure 4). Pathway analysis showed that cell survival related pathways regulated by TLN contained ErbB signaling pathway, neurotrophin signaling pathway, and phosphatidylinositol signaling system (Table 1). These results indicated that TLN may exert protective effects against morphological changes of sciatic nerve through regulating these genes of above pathways, such as Nrg1, Pik3cb, Mtor, and Gabl (Tables 1 and 2).

3.2.2. TLN Upregulates Gene Expression of Nrg1, Mtor, and Gab1 in Sciatic Nerve of Diabetic Rats. Cell-survival genes (Nrg1, Mtor, Gabl, and Pi3kcb) identified from the microarray analysis were selected and performed qPCR for validation. Results showed that the TLN intervention upregulated the Nrg1, Mtor, and Gabl expression levels, while the expression pattern of Pi3kcb were not consistent with the microarray data (Table 2 and Figure 5). Since Pi3kcb is the crucial gene of the Neurotrophin signaling pathway and phosphatidylinositol signaling system, there was no sense to do deeper study the two pathways.

3.2.3. TLN Upregulates Expression of P-ErbB2, P-Erk, and PBad (Ser112) in Sciatic Nerve of Diabetic Rat. In brief, we
TABLE 1: Cell survival-related pathways regulated by TLN.

\begin{tabular}{lcc}
\hline Pathway name & $\begin{array}{c}\text { Positive and } \\
\text { negative genes }\end{array}$ & $\begin{array}{c}\text { Enrichment } \\
\text { test } P \text { value }\end{array}$ \\
\hline & Nrg1 & \\
ErbB signaling pathway & Pik3cb & 0.0121 \\
& Gab1 & \\
& Mtor & \\
Neurotrophin signaling pathway & Pik3cb & $5.0 E-4$ \\
Phosphatidylinositol signaling system & Gab1 & \\
\hline
\end{tabular}

Enrichment test $P$ value $(P<0.05)$ means significant. Some genes listed are involved in more than one pathway. Nrg1: Neuregulin 1; Mtor: Mammalian target of rapamycin; Gab1: GRB2-associated binding protein 1 .

focused on Nrg1/ErbB2 pathway and do further validation by western blot. It is demonstrated that there was little variation of total ErbB2, Erk, Bad, Akt, and P-Akt after STZ injection $(P>0.05)$, and the phosphor-Bad (Ser136) cannot be detected by western blot. Likewise, phosphorylated levels of ErbB2, Erk, and Bad (Ser112) declined 3.6-fold, 1.9-fold, 4.4fold in the STZ group (no intervention after STZ injection) when compared with the control group, respectively. In contrast, the expression levels of phosphorylated protein were similar to normal rat with TLN and $\alpha$-LA intervention after STZ injection (Figure 6).

Taken together, these results from western blot and qPCR demonstrated that TLN could enhance NRG/ErbB2 $\rightarrow$ Erk/Bad dependent SCs-survival signal pathway (Figures 5 and 6). 


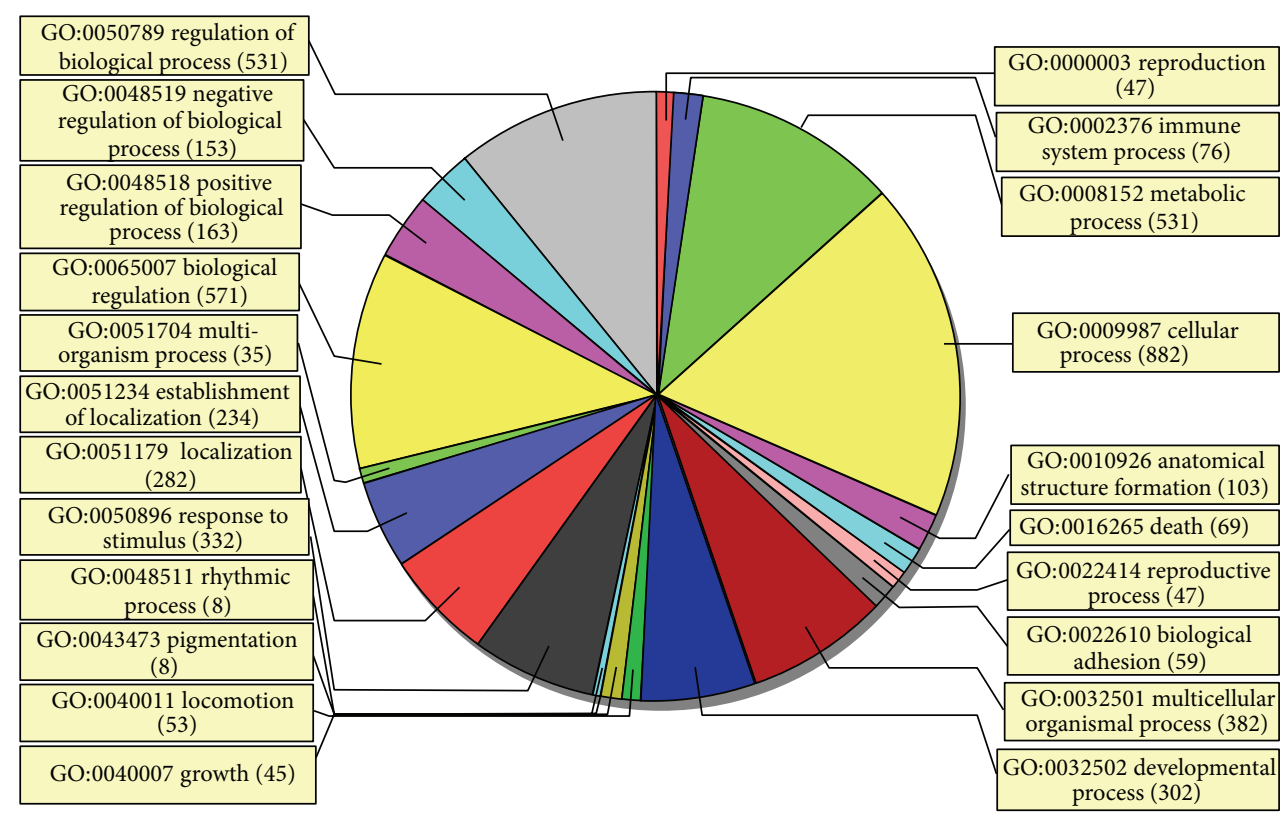

(a)

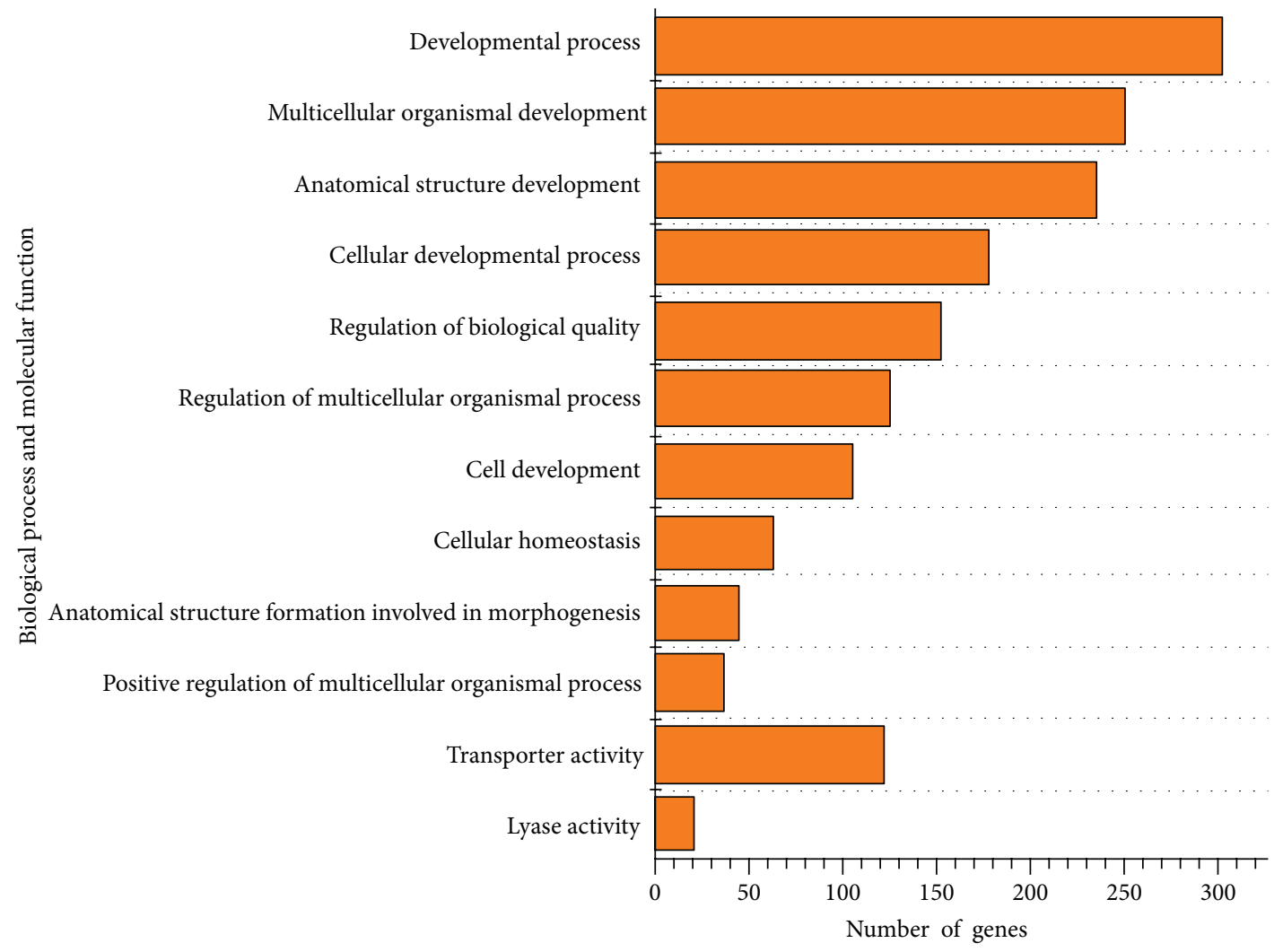

(b)

FIGURE 4: GO analysis and significantly altered categories regulated by TLN. (a) Gene ontology category of biological process for differentially expressed genes regulated by TLN. (b) The significant GO categories for differently expressed genes. The vertical axis represents the GO category, and the horizontal axis represents the number of genes changed in each category. There are 12 categories that were significantly altered (enrichment $P$ value $<0.05$ and number of changed genes $>20$ ). The first 10 categories are involved in biological process; the last two are involved in molecular function. GO: gene ontology. 
TABLE 2: Major genes in cell survival-related pathways upregulated by TLN.

\begin{tabular}{|c|c|c|c|c|c|c|}
\hline Probe Id & Gene Id & Gene symbol & Gene name & $\begin{array}{c}\text { FC: STZ } \\
\text { versus CTL }\end{array}$ & $\begin{array}{c}\text { FC: TLN } \\
\text { versus STZ }\end{array}$ & $\begin{array}{c}\text { FC: } \alpha \text {-LA } \\
\text { versus STZ }\end{array}$ \\
\hline A_44_P438143 & 112400 & Nrg1 & Neuregulin 1 & 0.3638 & 2.2264 & 2.1083 \\
\hline A_43_P12820 & 85243 & Pik3cb & $\begin{array}{c}\text { Phosphoinositide-3-kinase, catalytic, and } \\
\text { beta polypeptide }\end{array}$ & 0.3298 & 3.3562 & 2.8317 \\
\hline A_44_P191924 & 56718 & Mtor & $\begin{array}{c}\text { Mechanistic target of rapamycin } \\
\text { (serine/threonine kinase) }\end{array}$ & 0.3786 & 2.2988 & 1.9853 \\
\hline A_44_P176342 & 361388 & Gab1 & GRB2-associated binding protein 1 & 0.2205 & 4.8388 & 3.8136 \\
\hline
\end{tabular}

FC: fold change.

\section{Discussion}

The present study provides direct evidence for a crucial role of TLN in function and morphology of sciatic nerve after diabetes. The protective effects of the compound recipe TLN may be attributed to promote SCs-survival through the activation of Nrg1/ErbB2 $\rightarrow$ Erk/Bad (Ser112) signal pathway by increasing the phosphorylation of ErbB2, Erk, and Bad (Ser112) at a protein level and mRNA expression of Nrg1, Mtor, and Gabl. This finding supports the hypothesis that TLN was a critical regulator in SCs survival and recovery of peripheral nerve regeneration ability. Recently there has been a great deal of interest in SCs due to the key role of it in myelination regeneration and repair after peripheral nerve injury.

DPN is a long-term common complication of diabetes. In this study, STZ was used to induce diabetic neuropathy model, which was widely performed in Sprague-Dawley rats [21], Wistar rats [27], and transgenic mice [28]. The STZ-induced diabetic rats beard the typical characteristics of DPN including demyelination and axial degeneration of sciatic nerve and reduction of peripheral nerve conduction velocity, which were consistent with published observations [29]. In addition, $\alpha$-LA was selected as a positive control in the present study because of its antioxidative activity [30, 31].

In fact, ErbB2 signal pathway was most closely linked to SCs survival among the three screened signal pathways by microarray (Table 1), and Nrg1, Mtor, and Gabl are key genes of ErbB2 signal pathway. Mtor is a conserved serine/threonine protein kinase and a member of the phosphatidylinositol 3-kinase-related kinase family. Mtor plays key roles in sensing nutrition signal, regulation of cell growth and proliferation [32], and regulation of insulin response by the insulin response element [33]. Meanwhile, due to the proapoptosis effect of c-Jun-NH2-terminal kinase pathway activation, Gabl has an indirect antiapoptosis effect. Biochemical analysis suggested Gabl played a positive role in coupling the signal relay between cytokine receptors and the Erk pathway [34]. More important, Nrg1, also known as glial growth factor, is mainly produced by SCs and transported by axons and plays a crucial role in SCs survival. Nrg1 belongs to Neuregulin gene family which contains four members termed as Nrg1, Nrg2, Nrg3, and Nrg4. Among them, Nrg1 is the most characteristic gene, and it is believed to be able to rescue SCs from apoptosis and act as a predominant survival factor during SCs maturation [35]. Recently studies also demonstrated that NRG signal provided trophic support to myelin-producing cells and contributed to myelination in peripheral nerve system and velocity of conduction $[36,37]$. In our study, these three genes discussed above appeared to be key factors through which TLN might promote SCs survival.

The binding and activation of Nrg1 and ErbB2/ErbB3 coreceptor to activate the downstream signal pathways is very important to transmit SCs survival signal. Being a family member of polypeptide growth/differentiation factors containing an epidermal growth factor-like motif, Nrg1 could activate membrane-associated ErbB tyrosine kinase receptors and mediate SCs differentiation, proliferation, and migration through binding and activation of a heterodimeric ErbB2/ErbB3 coreceptor [38]. ErbB2 itself does not bind to NRG, while ErbB3 alone could bind to NRG but could not transmit signals. Since it is necessary for ErbB2 and ErbB3 to form a high affinity coreceptor complex to transmit SCs survival signal, the expression level of P-ErbB2 could reflect NRG receptor downstream signal intensity. In this study, the expression level of P-ErbB2 decreased after STZ induction, suggesting that there was a close relationship between Nrg1ErbB2 signal transduction and sciatic nerve morphology change/conduction velocity. These data contradict with some reports [39] but agree with some others [40-42]. The underlying causes for this discrepancy are unknown. The present study also showed that TLN enhanced NRG receptor signal intensity through increasing Nrg1 mRNA expression and phosphorylation of ErbB2 at a protein level after peripheral nerve injury in STZ-R.

In addition, after binding to the high-affinity ErbB2/ErbB3 coreceptor complex, Nrg could potentially activate several distinct signaling pathways, mainly including p21Ras/Raf-1/MEK/Erk cascade [43] and PI3K/Akt signaling pathway [44]. The phosphorylation of bad either on serine 112 by Erk or serine 136 by Akt promotes its dissociation from the cytosolic protein 14-3-3, leading to the release of $\mathrm{Bcl}-2$ and Bcl-xl to inhibit SCs apoptosis. Obviously, the phosphorylation of bad is the key link for TLN to promote SCs survival. One study showed that NRG-ErbB2 receptor signaling promoted SCs survival through a downstream $\mathrm{PI} 3 \mathrm{~K} / \mathrm{Akt} / \mathrm{Bad}$ pathway [41]. In this study, we detected that the significant change of phospho-Erk and phospho-Bad (Ser112), accompanying insignificant variation of phosphoAkt and no detection of phospho-Bad at the ser 136 residue, 


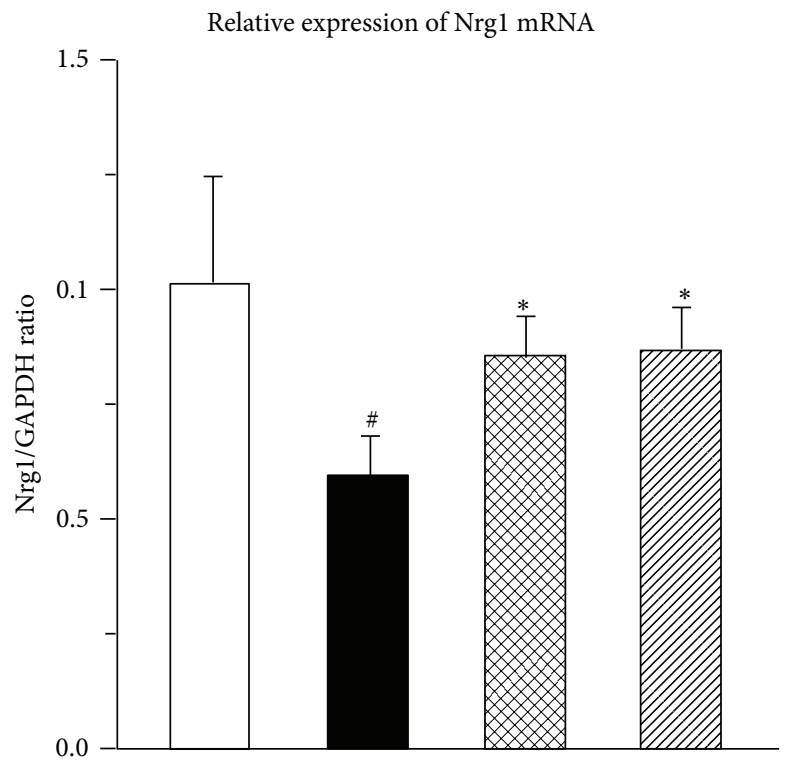

(a)

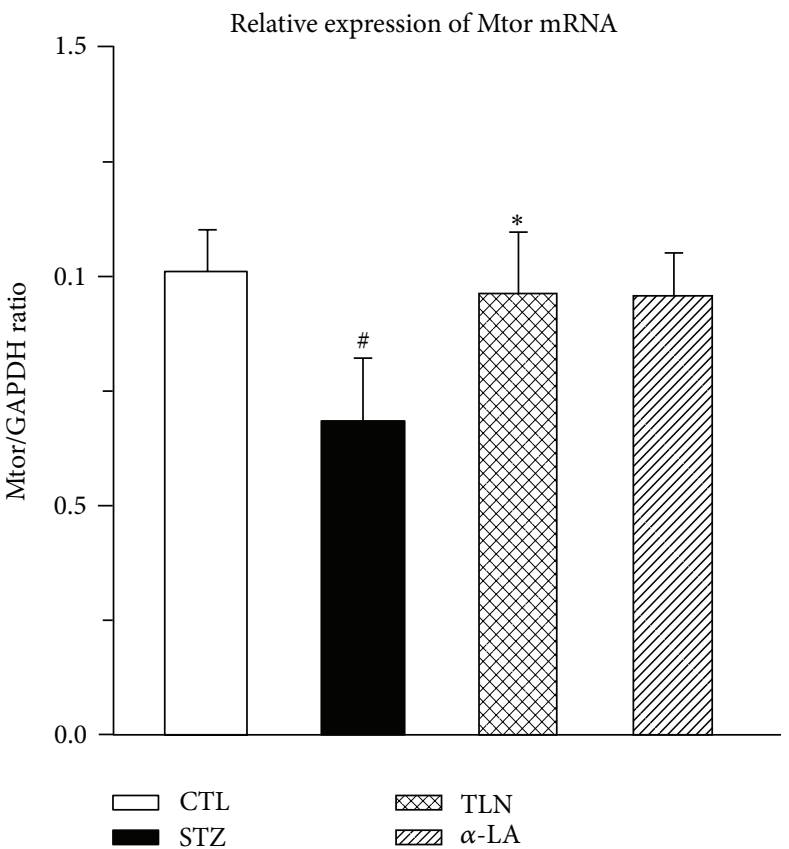

(c)

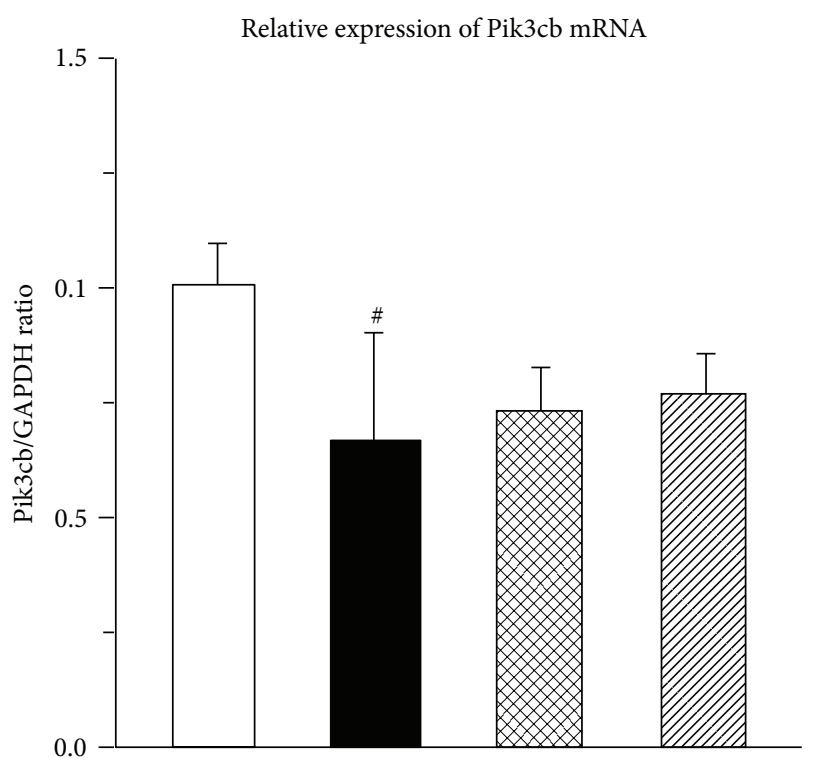

(b)

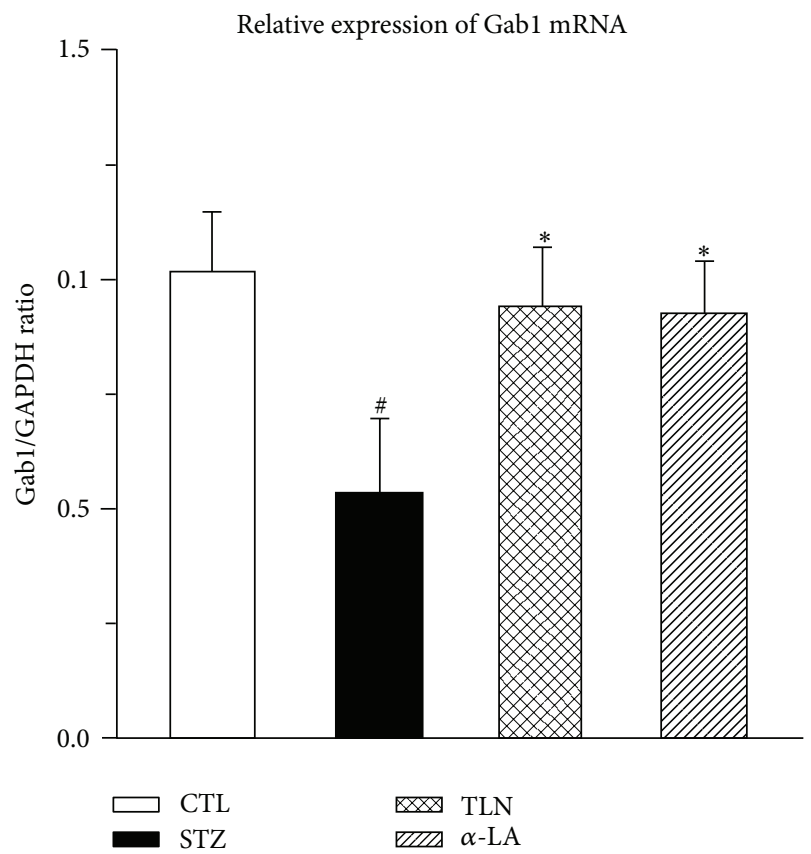

(d)

FIGURE 5: Effect of TLN and $\alpha$-LA on cell survival related genes in sciatic nerve of streptozotocin-induced rats. Relative expression level change $\left(2^{-\Delta \Delta C T}\right)$ of 4 genes in qPCR, (a) Nrgl, (b) Pik3cb, (c) Mtor, and (d) Gabl. Statistical analysis was performed using the ANOVA procedure $t$ test (LSD). Data are expressed as mean \pm SD. ${ }^{\#} P<0.05$, when compared with CTL group; ${ }^{*} P<0.05$, when compared with STZ group. The mRNA expression of Nrg1, Pik3cb, Mtor, and Gabl decreased compared with CTL group $(P<0.05)$; TLN intervention could increase the expression level of Nrg1, Mtor, and Gabl compared with STZ group $(P<0.05)$. The alpha-lipoic acid intervention trend was consistent with Tang-Luo-Ning except Gabl. There were no significant differences of Pik3cb mRNA between the three groups (STZ, $\alpha$-LA, and TLN). Four groups: CTL group: nonstreptozotocin-induced group, STZ group: streptozotocin-induced diabetic group, TLN group: Tang-Luo-Ning group, and $\alpha$-LA group: alpha-lipoic acid group. ANOVA: analysis of variance; LSD- $t$ test: least significant difference $t$ test; qPCR: quantitative real time polymerase chain reaction. 


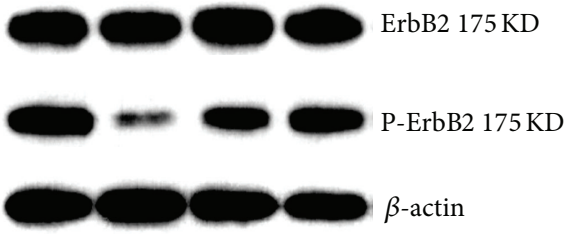

(a)

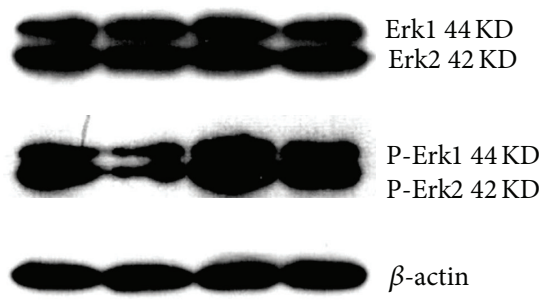

(c)

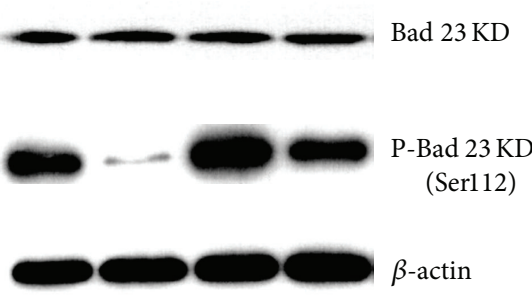

(e)

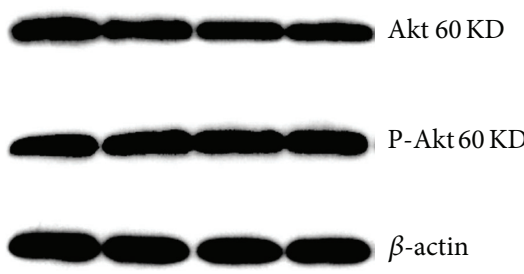

(g)

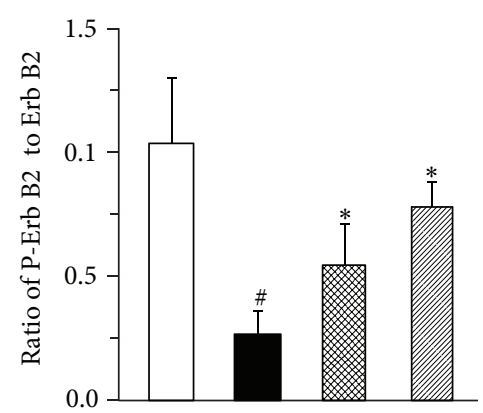

(b)

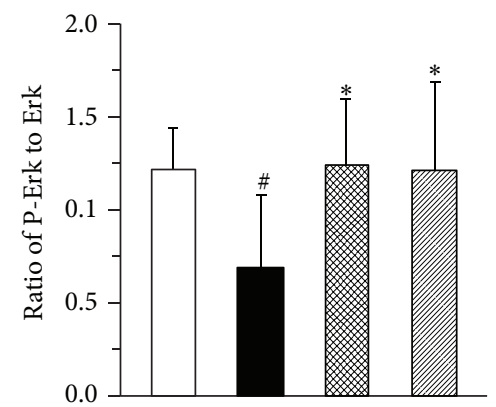

(d)

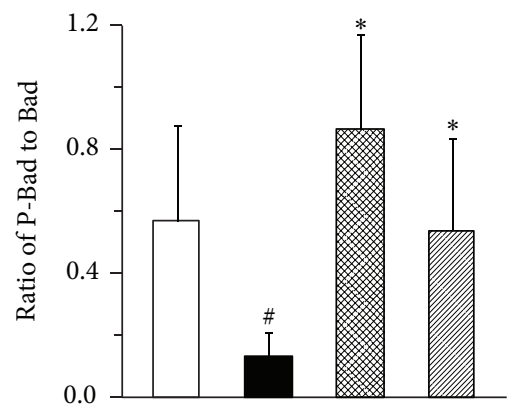

(f)

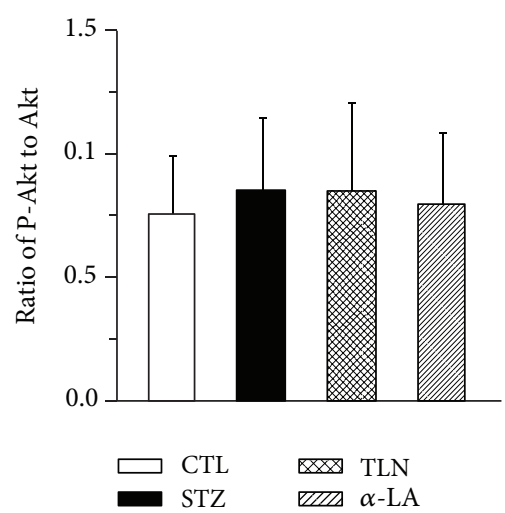

(h)

FIGURE 6: Effect of TLN and $\alpha$-LA on Nrg1/ErbB2 downstream signal pathway in sciatic nerve of streptozotocin-induced rats. A representative western blot ((a), (c), (e), and (g)) and the grey value analyses of protein expression ((b), (d), (f), and (h)) in sciatic nerves from different experimental groups. ((a), (b)) Ratio of P-ErbB2 to T-ErbB2 (P/T); ((c), (d)) ratio of P-Erk to T-Erk (P/T); ((e), (f)) ratio of P-Bad (Ser112) to T-Bad (P/T); ((g), (h)) ratio of P-Akt to T-Akt (P/T). Statistical analysis was performed using the ANOVA procedure $t$ test (LSD). Data are expressed as mean $\pm \mathrm{SD} .{ }^{\#} P<0.05$ versus CTL group, ${ }^{*} P<0.05$ versus STZ group. $n=4$ per group. The protein expression of ratio of ErbB2 Erk, Bad $(\mathrm{P} / \mathrm{T})$ decreased compared with CTL group $(P<0.05)$, while Tang-Luo-Ning and alpha-lipoic acid intervention could increase the ratio above compared with STZ group $(P<0.05)$. There were no significant differences of ratio of Akt $(\mathrm{P} / \mathrm{T})$ between the four groups. Four groups: CTL group: nonstreptozotocin-induced group, STZ group: streptozotocin-induced diabetic group, TLN group: Tang-Luo-Ning group, and $\alpha$-LA group: alpha-lipoic acid group. ANOVA: analysis of variance; LSD- $t$ test: least significant difference $t$ test. 
suggesting that SCs-survival effect by TLN could be due to the Erk/Bad (Ser112) signal but not the PI3K/Akt/Bad (Ser136) signal.

Despite a marked improvement in the function and morphology of sciatic nerve with TLN intervention after STZ injection, administration of TLN did not completely halt the progression of sciatic nerve injury. This suggests that there may be other signaling pathways involved in pathogenesis of nerve damage. Furthermore, although the Nrg1/ErbB2 receptor dependent SCs-survival effect of TLN is explicit in this in vivo animal model, its biologic relevance in vitro remains unclear. It will be important to evaluate the therapeutic role of TLN in future in vitro studies.

\section{Conclusion}

In conclusion, the findings of the current study provide support to the hypothesis that compound recipe TLN could inhibit SC apoptosis, which lead to an improvement in sciatic MNCV, distal digital SNCV and sciatic nerve pathological injury, suggesting TLN offering a promising alternative medicine for the DPN patients to delay the progression. The mechanism of the protective effect of TLN on promoting SCs survival may be partly due to activate $\mathrm{Nrg1/ErbB2} \rightarrow$ Erk/bad signal pathway.

\section{Conflict of Interests}

The authors declare that they have no conflict of interests, financial or otherwise.

\section{Acknowledgments}

This work was supported by National Natural Science Foundation of China (NSFC) (no. 30873253). Thus, the authors thank NSFC for financial support. The authors also thank Shanghai Biotechnology Co., Ltd for providing technical assistance related to the study.

\section{References}

[1] S. Tesfaye and D. Selvarajah, "Advances in the epidemiology, pathogenesis and management of diabetic peripheral neuropathy," Diabetes/Metabolism Research and Reviews, vol. 28, supplement 1, pp. 8-14, 2012.

[2] A. J. Boulton, A. I. Vinik, J. C. Arezzo et al., "Diabetic neuropathies: a statement by the American Diabetes Association," Diabetes Care, vol. 28, no. 4, pp. 956-962, 2005.

[3] P. J. Oates, "Polyol pathway and diabetic peripheral neuropathy," International Review of Neurobiology, vol. 50, pp. 325-328, 2002.

[4] J. L. Wautier and P. J. Guillausseau, "Diabetes, advanced glycation endproducts and vascular disease," Vascular Medicine, vol. 3, no. 2, pp. 131-137, 1998.

[5] K. J. Way, N. Katai, and G. L. King, "Protein kinase C and the development of diabetic vascular complications," Diabetic Medicine, vol. 18, no. 12, pp. 945-959, 2001.

[6] T. Purves, A. Middlemas, S. Agthong et al., "A role for mitogenactivated protein kinases in the etiology of diabetic neuropathy," The FASEB Journal, vol. 15, no. 13, pp. 2508-2514, 2001.
[7] M. Brownlee, "The pathobiology of diabetic complications: a unifying mechanism," Diabetes, vol. 54, no. 6, pp. 1615-1625, 2005.

[8] A. M. Schmidt, S. D. Yan, J. L. Wautier, and D. Stern, "Activation of receptor for advanced glycation end products: a mechanism for chronic vascular dysfunction in diabetic vasculopathy and atherosclerosis," Circulation Research, vol. 84, no. 5, pp. 489497, 1999.

[9] J. Yu, Y. Zhang, S. Sun et al., "Inhibitory effects of astragaloside IV on diabetic peripheral neuropathy in rat," Canadian Journal of Physiology and Pharmacology, vol. 84, no. 6, pp. 579-587, 2006.

[10] L. Q. Sun, B. Xue, X. J. Li et al., "Inhibitory effects of salvianolic acid B on apoptosis of Schwann cells and its mechanism induced by intermittent high glucose," Life Sciences, vol. 90, no. 3-4, pp. 99-108, 2012.

[11] L. Q. Sun, Y. Y. Chen, X. Wang et al., “The protective effect of alpha lipoic acid on Schwann cells exposed to constant or intermittent high glucose," Biochemical Pharmacology, vol. 84, no. 7, pp. 961-973, 2012.

[12] L. Eckersley, "Role of the Schwann cell in diabetic neuropathy," International Review of Neurobiology, vol. 50, pp. 293-321, 2002.

[13] L. Stenberg, M. Kanje, K. Dolezal, and L. B. Dahlin, "Expression of activating transcription factor 3 (ATF 3 ) and caspase 3 in Schwann cells and axonal outgrowth after sciatic nerve repair in diabetic BB rats," Neuroscience Letters, vol. 515, no. 1, pp. 3438, 2012.

[14] A. Krishnan, "Neuregulin-1 type I: a hidden power within Schwann cells for triggering peripheral nerve remyelination," Science Signaling, vol. 6, no. 270, article jc1, 2013.

[15] D. S. Skundric and R. P. Lisak, "Role of neuropoietic cytokines in development and progression of diabetic polyneuropathy: from glucose metabolism to neurodegeneration," Experimental Diabesity Research, vol. 4, no. 4, pp. 303-312, 2003.

[16] Q. L. Wu and X. C. Liang, "Survey of current experimental studies of effects of traditional Chinese compound recipe on diabetic peripheral neuropathy," Zhongguo Zhong Yao Za Zhi, vol. 32, no. 9, pp. 775-778, 2007.

[17] Y. B. Gao, R. H. Liu, X. C. Yu et al., "Clinical observation on patients of diabetic peripheral nervous lesion," Journal of Beijing University of TCM, vol. 20, no. 4, pp. 50-53, 1997.

[18] H. Zhou, Y. B. Gao, T. H. Liu et al., "Clinical trial of tangluoning for treating diabetic peripheral neuropathy," Journal of Beijing University of TCM, vol. 25, no. 4, pp. 59-62, 2002.

[19] W. L. Li, H. C. Zheng, J. Bukuru, and N. de Kimpe, "Natural medicines used in the traditional Chinese medical system for therapy of diabetes mellitus," Journal of Ethnopharmacology, vol. 92, no. 1, pp. 1-21, 2004.

[20] Q. Qin, J. Niu, Z. Wang, W. Xu, Z. Qiao, and Y. Gu, "Astragalus membranaceus inhibits inflammation via phosphoP38 mitogen-activated protein kinase (MAPK) and nuclear factor (NF) $\kappa \mathrm{B}$ pathways in advanced glycation end productstimulated macrophages," International Journal of Molecular Sciences, vol. 13, no. 7, pp. 8379-8387, 2012.

[21] J. Yu, Y. Zhang, S. Sun et al., "Inhibitory effects of astragaloside IV on diabetic peripheral neuropathy in rats," Canadian Journal of Physiology and Pharmacology, vol. 84, no. 6, pp. 579-587, 2006.

[22] Y. B. Gao, Z. Y. Zhu, D. W. Zou, B. M. Li, and J. S. Peng, "Effect of TangluoNing on cell apoptosis of dorsal root ganglion in diabetic rats," Chinese Journal of Basic Medicine in Traditional Chinese Medicine, vol. 18, no. 6, pp. 616-618, 2012. 
[23] D. W. Zou, Z. Y. Zhu, and Y. B. Gao, "Effects of traditional Chinese medicine on oxidative stress and ultrastructure of diabetic rats' sciatic nerve," China Journal of Traditional Chinese Medicine and Pharmacy, vol. 27, no. 5, pp. 1253-1256, 2012.

[24] M. J. Stevens, I. Obrosova, X. Cao, C. van Huysen, and D. A. Greene, "Effects of DL-alpha-lipoic acid on peripheral nerve conduction, blood flow, energy metabolism, and oxidative stress in experimental diabetic neuropathy," Diabetes, vol. 49, no. 6, pp. 1006-1015, 2000.

[25] M. W. Pfaffl, "A new mathematical model for relative quantification in real-time RT-PCR," Nucleic Acids Research, vol. 29, no. 9, article e45, 2001.

[26] N. E. Cameron, M. A. Cotter, D. H. Horrobin, and H. J. Tritschler, "Effects of alpha-lipoic acid on neurovascular function in diabetic rats: interaction with essential fatty acids," Diabetologia, vol. 41, no. 4, pp. 390-399, 1998.

[27] S. S. Kamboj, R. K. Vasishta, and R. Sandhir, "N-acetylcysteine inhibits hyperglycemia-induced oxidative stress and apoptosis markers in diabetic neuropathy," Journal of Neurochemistry, vol. 112, no. 1, pp. 77-91, 2010.

[28] R. Stavniichuk, H. Shevalye, H. Hirooka, J. L. Nadler, and I. G. Obrosova, "Interplay of sorbitol pathway of glucose metabolism, 12/15-lipoxygenase, and mitogen-activated protein kinases in the pathogenesis of diabetic peripheral neuropathy," Biochemical Pharmacology, vol. 83, no. 7, pp. 932-940, 2012.

[29] S. Yagihashi, M. Kamijo, and K. Watanabe, "Reduced myelinated fiber size correlates with loss of axonal neurofilaments in peripheral nerve of chronically streptozotocin diabetic rats," The American Journal of Pathology, vol. 136, no. 6, pp. 1365-1373, 1990.

[30] M. Nagamatsu, K. K. Nickander, J. D. Schmelzer et al., "Lipoic acid improves nerve blood flow, reduces oxidative stress, and improves distal nerve conduction in experimental diabetic neuropathy," Diabetes Care, vol. 18, no. 8, pp. 1160-1167, 1995.

[31] Z. G. Xu, C. G. Wu, L. Wang, Y. N. Chen, and Y. Chen, "Effects of alpha lipoic acid on oxidative stress and apoptosis of sciatic nerve in diabetic rat," Journal of Jiangsu University, vol. 21, no. 1, pp. 15-17, 2011.

[32] E. Buck, A. Eyzaguirre, J. D. Haley, N. W. Gibson, P. Cagnoni, and K. K. Iwata, "Inactivation of Akt by the epidermal growth factor receptor inhibitor erlotinib is mediated by HER-3 in pancreatic and colorectal tumor cell lines and contributes to erlotinib sensitivity," Molecular Cancer Therapeutics, vol. 5, no. 8, pp. 2051-2059, 2006.

[33] C. Taha, Z. Liu, J. Jin, H. Al-Hasani, N. Sonenberg, and A. Klip, "Opposite translational control of GLUT1 and GLUT4 glucose transporter mRNAs in response insulin. Role of mammalian target of rapamycin, protein kinase $\mathrm{B}$, and phosphatidylinositol 3-kinase in GLUT1 mRNA translation," The Journal of Biological Chemistry, vol. 274, no. 46, pp. 33085-33091, 1999.

[34] Y. Sun, J. Yuan, H. Liu et al., "Role of Gabl in UV-induced c-Jun NH2-terminal kinase activation and cell apoptosis," Molecular and Cellular Biology, vol. 24, no. 4, pp. 1531-1539, 2004.

[35] Z. Ma, J. Wang, F. Song, and J. A. Loeb, "Critical period of axoglial signaling between neuregulin-1 and brain-derived neurotrophic factor required for early Schwann cell survival and differentiation," Journal of Neuroscience, vol. 31, no. 26, pp. 9630-9640, 2011.

[36] G. V. Michailov, M. W. Sereda, B. G. Brinkmann et al., "Axonal neuregulin-1 regulates myelin sheath thickness," Science, vol. 304, no. 5671, pp. 700-703, 2004.
[37] C. Taveggia, G. Zanazzi, A. Petrylak et al., "Neuregulin-1 type III determines the ensheathment fate of axons," Neuron, vol. 47, no. 5, pp. 681-694, 2005.

[38] S. Heermann, J. Schmücker, U. Hinz et al., "Neuregulin 1 type III/ErbB signaling is crucial for schwann cell colonization of sympathetic axons," PLoS ONE, vol. 6, no. 12, Article ID e28692, 2011.

[39] J. F. McGuire, S. Rouen, E. Siegfreid, D. E. Wright, and R. T. Dobrowsky, "Caveolin-1 and altered neuregulin signaling contribute to the pathophysiological progression of diabetic peripheral neuropathy," Diabetes, vol. 58, no. 11, pp. 2677-2686, 2009.

[40] S. Chen, M. O. Velardez, X. Warot et al., "Neuregulin 1-erbB signaling is necessary for normal myelination and sensory function," Journal of Neuroscience, vol. 26, no. 12, pp. 3079-3086, 2006.

[41] Y. Li, G. I. Tennekoon, M. Birnbaum, M. A. Marchionni, and J. L. Rutkowski, "Neuregulin signaling through a PI3K/Akt/Bad pathway in Schwann cell survival," Molecular and Cellular Neuroscience, vol. 17, no. 4, pp. 761-767, 2001.

[42] J. M. Newbern, X. Li, S. E. Shoemaker et al., "Specific functions for ERK/MAPK signaling during PNS development," Neuron, vol. 69, no. 1, pp. 91-105, 2011.

[43] J. Newbern and C. Birchmeier, "Nrg1/ErbB signaling networks in Schwann cell development and myelination," Seminars in Cell and Developmental Biology, vol. 21, no. 9, pp. 922-928, 2010.

[44] Z. Liu, H. Jiang, H. Li, H. Liu, X. Xu, and Z. Li, “The effects of neuregulin-1beta on neuronal phenotypes of primary cultured dorsal root ganglion neurons by activation of PI3K/Akt," Neuroscience Letters, vol. 511, no. 1, pp. 52-57, 2012. 


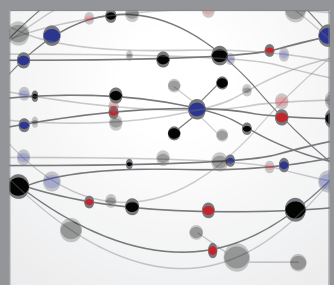

The Scientific World Journal
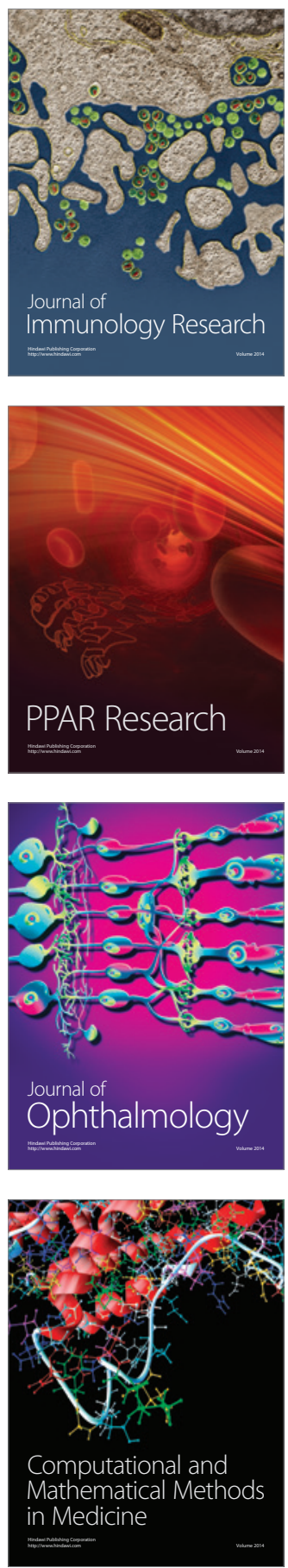

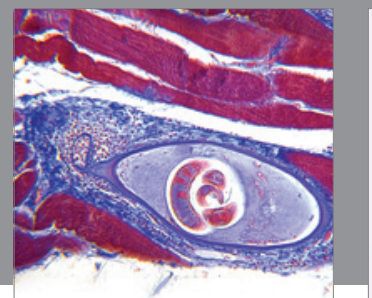

Gastroenterology

Research and Practice
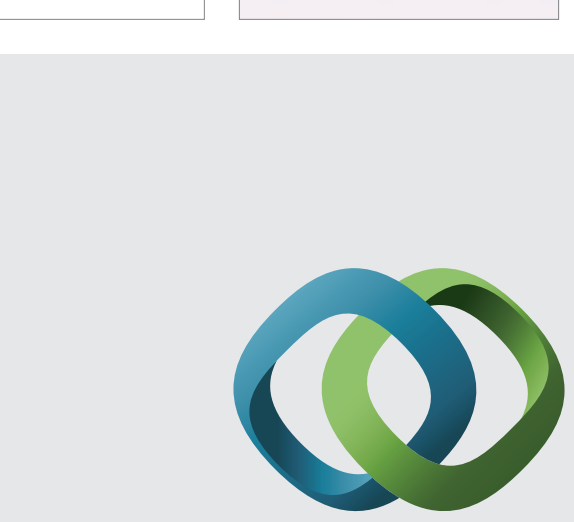

\section{Hindawi}

Submit your manuscripts at

http://www.hindawi.com
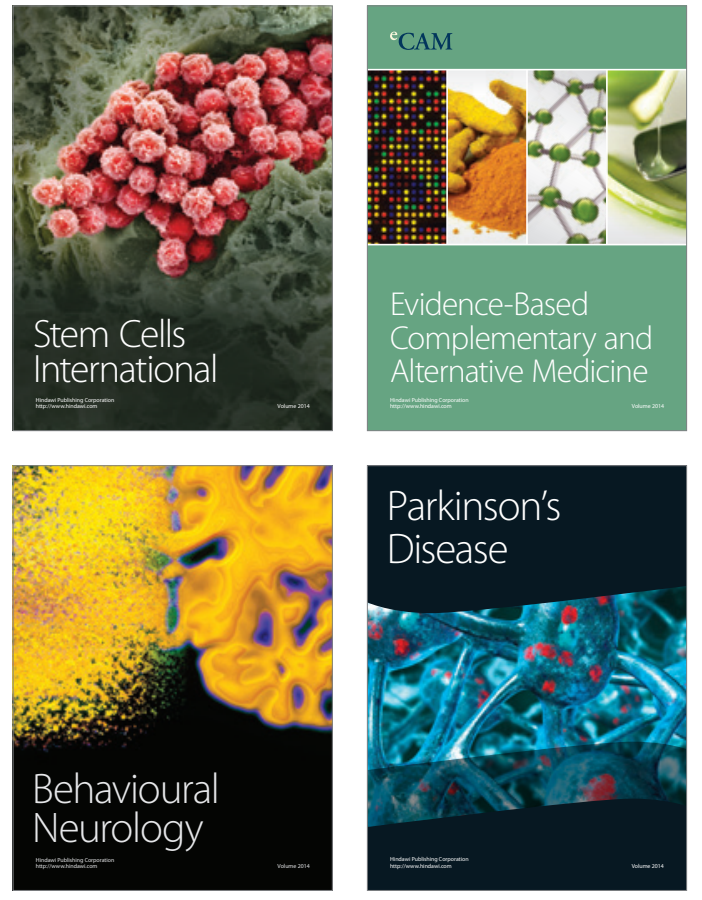
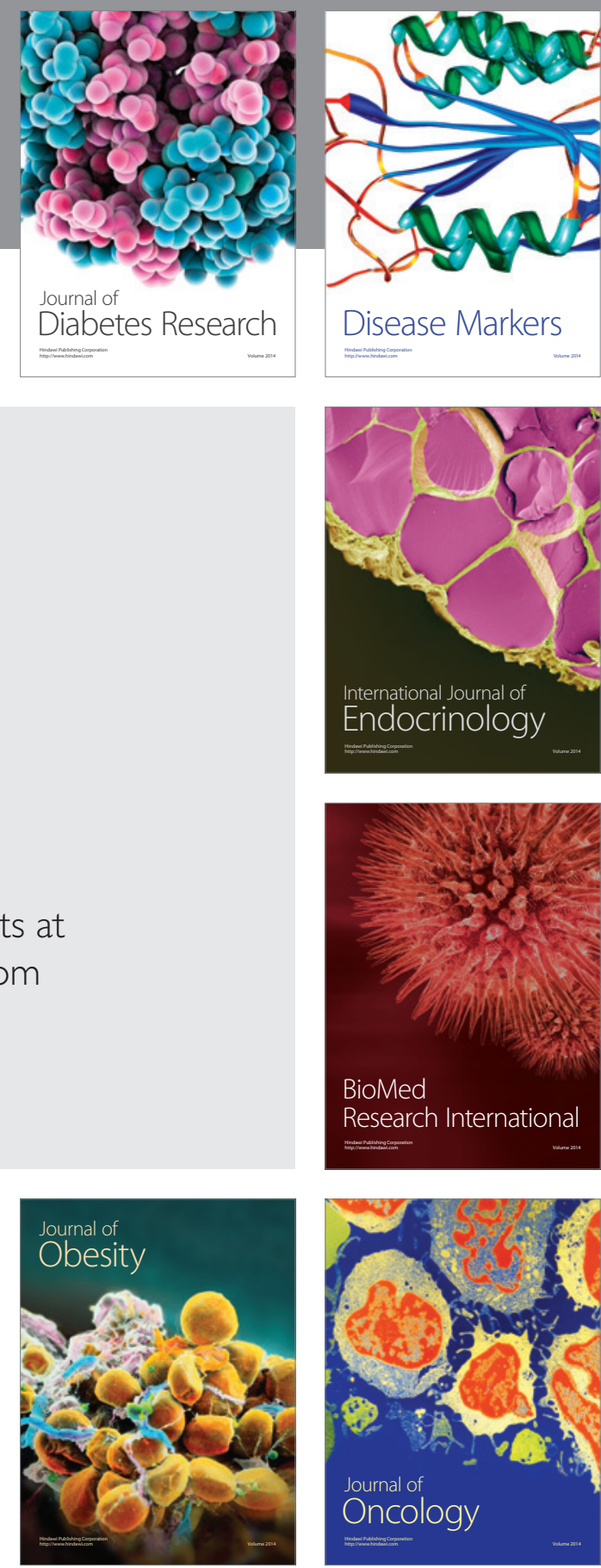

Disease Markers
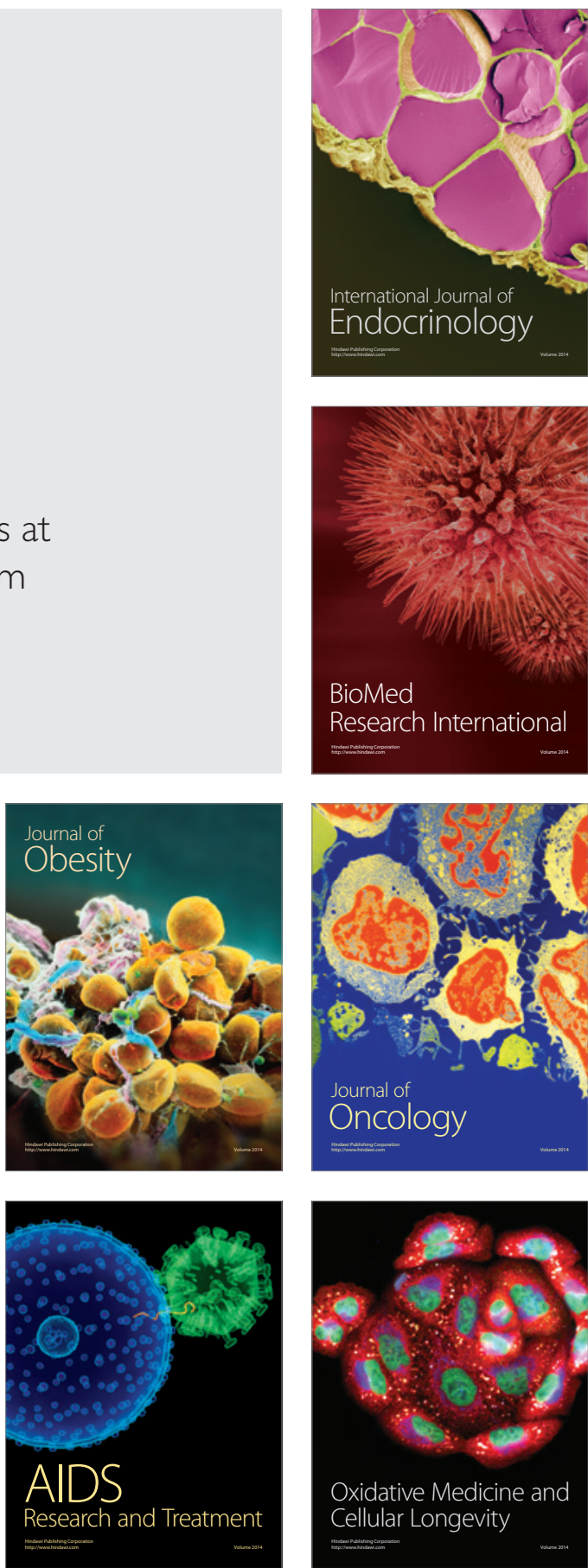